\title{
A VERY GENERAL THEOREM ON SYSTEMS OF DISTINCT REPRESENTATIVES
}

\author{
BY \\ RICHARD A. BRUALDI $\left({ }^{1}\right)$
}

1. Introduction. Currently the theory of systems of distinct representatives (and the closely allied theory of transversals) is being carefully examined and reworked, often in a more general context which allows for the transfinite situation. This theory can be said to have had its beginning in 1935 when P. Hall proved his now celebrated theorem for the existence of a system of distinct representatives of a finite family of sets. In a no less significant paper M. Hall, Jr. (in 1948) extended P. Hall's theorem to infinite families of finite sets. Around these two theorems a considerable literature has grown (for an excellent survey and thorough bibliography see [10]). The two theorems have been refined in various ways by requiring that the system of distinct representatives have additional properties. It is however true that these refinements can be obtained by applying the original theorems to a modified family of sets. For finite families this is implicit in the work of Ford and Fulkerson [3] who show how most of these refinements can be obtained from their maximum flow-minimum cut theorem for flows in networks. For finite or infinite families Mirsky and Perfect [10], [11] have shown how these refinements can be obtained from the original theorems of the two Halls and a generalization of a mapping theorem of Banach [1]. In a recent paper [2] we obtained a further generalization of Banach's mapping theorem. This theorem along with M. Hall's theorem enables us to prove a very general theorem on systems of distinct representatives, which is in fact a transfinite and symmetrized form of a theorem of A. J. Hoffman and H. W. Kuhn. The theorem we prove contains as special cases (that is, without further refinement) all theorems that we know which assert the existence of a system of distinct representatives of a given family of sets or subfamily thereof with certain properties being required. We then can prove a theorem giving necessary and sufficient conditions that a family of sets possess a family of subsets whose cardinalities lie within prescribed bounds and where the frequencies of occurrences in these subsets of the elements lie within prescribed bounds. This will be made more precise later. From this we also obtain an extension to locally finite graphs of Ore's solution [12] of the so-called "subgraph problem for directed graphs" and for that matter a generalization of Ore's solution due to Ford and Fulkerson [3].

Received by the editors April 9, 1968.

(1) The research of the author was partially supported by NSF Grant No. GP-7073. 
2. Preliminaries. Let $E$ be an arbitrary set and $\mathfrak{A}(I)=\left(A_{i}: i \in I\right)$ a family of subsets of $E$ indexed by a set $I$. Thus, although $i \neq i^{\prime}$, it may be that $A_{i}=A_{i^{\prime}}$. If $I^{\prime} \subseteq I$, then $\mathfrak{A}\left(I^{\prime}\right)=\left(A_{i}: i \in I^{\prime}\right)$, a subfamily of $\mathfrak{A}(I)$. A family $\left(x_{j}: j \in J\right)$ is a system of representatives of $\mathfrak{A}(I)$ if there is a bijection $\sigma: J \rightarrow I$ such that $x_{j} \in A_{\sigma(j)}$ for all $j \in J$. If in addition the $x_{j}$ are distinct, then $\left(x_{j}: j \in J\right)$ is a system of distinct representatives of $\mathfrak{A}(I)$. The subset $\left\{x_{j}: j \in J\right\}$ of $E$ is then called a transversal of $\mathfrak{A}(I)$. Thus a subset $E^{0}$ of $E$ is a transversal of $\mathfrak{A}(I)$ if and only if the elements of $E^{0}$ can be indexed by $I,\left(x_{i}: i \in I\right)$, in such a way that $x_{i} \in A_{i}$ for all $i \in I$. We shall usually phrase our results in terms of transversals rather than systems of distinct representatives.

With a given family $\mathfrak{A}(I)=\left(A_{i}: i \in I\right)$ of subsets of a set $E$ we associate a dual family $\overline{\mathfrak{A}}(E)=\left(\bar{A}_{e}: e \in E\right)$ of subsets of $I$ indexed by $E$. For $e \in E, \bar{A}_{e}$ is defined by

$$
\bar{A}_{e}=\left\{i \in I: e \in A_{i}\right\} \text {. }
$$

The dual of the family $\overline{\mathfrak{A}}(E)$ is the original family so that each determines the other. Note that for $e \in E, \bar{A}_{e}$ is finite if and only if $e$ is a member of only finitely many $A$ 's.

The cardinality of a set $X$ is denoted by $|X|$. If $X$ and $Y$ are sets, then $X \backslash Y$ is the set of those elements of $X$ which are not elements of $Y$. For $X$ a set, $\sum_{k \in K} X_{k}$ is a partition of $X$ means $X=\bigcup_{k \in K} X_{k}$ and $X_{k} \cap X_{k^{\prime}}=\varnothing$ whenever $k \neq k^{\prime}$.

The following two theorems will be crucial in what follows.

THEOREM 1. The family $\mathfrak{A}(I)=\left(A_{i}: i \in I\right)$ of finite subsets of a set $E$ has a transversal (or, equivalently, a system of distinct representatives) if and only if for every finite subset $U$ of $I$

$$
\left|\bigcup_{i \in U} A_{i}\right| \geqq|U| .
$$

In case $|I|<\infty$ it is not necessary that the A's be finite sets.

The theorem for arbitrary index sets $I$ is due to M. Hall, Jr. [4]. For $|I|<\infty$, the theorem is the original one of P. Hall [5].

The second theorem that we shall need is a generalization of Banach's mapping theorem. We state it in the language of the present paper.

THEOREM 2. Let $\mathfrak{A}(I)=\left(A_{i}: i \in I\right)$ be a family of arbitrary subsets of a set $E$. Let $\sum_{k \in K} I_{k}$ and $\sum_{j \in J} E_{j}$ be partitions of I and $E$ respectively. Suppose for each $k \in K$ there are specified integers $c_{k}$ and $c_{k}^{\prime}$ with $0 \leqq c_{k} \leqq c_{k}^{\prime}$, and that for each $j \in J$ there are specified integers $d_{j}$ and $d_{j}^{\prime}$ with $0 \leqq d_{j} \leqq d_{j}^{\prime}$. If there is a subfamily $\mathfrak{A}\left(I^{\prime}\right)$ which has a transversal $E^{\prime}$ with

$$
\begin{aligned}
& c_{k} \leqq\left|I_{k}\right| I^{\prime} \mid \quad(k \in K) \\
& \left|E_{j}\right| E^{\prime} \mid \leqq d_{j}^{\prime} \quad(j \in J),
\end{aligned}
$$

and if there is a subfamily $\mathfrak{A}\left(I^{\prime \prime}\right)$ which has a transversal $E^{\prime \prime}$ with

$$
\begin{aligned}
& \left|I_{k}\right| I^{\prime \prime} \mid \leqq c_{k}^{\prime} \quad(k \in K) \\
& d_{j} \leqq\left|E_{j} \backslash E^{\prime \prime}\right| \quad(j \in J),
\end{aligned}
$$


then there exists a subfamily $\mathfrak{A}\left(I^{0}\right)$ which has a transversal $E^{0}$ with

$$
\begin{array}{ll}
c_{k} \leqq\left|I_{k}\right| I^{0} \mid \leqq c_{k}^{\prime} & (k \in K) \\
d_{j} \leqq\left|E_{j}\right| E^{0} \mid \leqq d_{j}^{\prime} & (j \in J) .
\end{array}
$$

It is permissible that the cardinalities $\left|I_{k}\right| I^{\prime} \mid$ and $\left|E_{j}\right| E^{\prime \prime} \mid$ be infinite. Theorem 2 is proved by the present author in [2].

3. The principal theorem. In [7] A. J. Hoffman and H. W. Kuhn prove a theorem which gives necessary and sufficient conditions for a finite family of subsets of a set $E$ to have a transversal which intersects a given partition of $E$ within prescribed bounds. The theorem below generalizes their theorem in two directions: (1) it makes the conclusion of the theorem symmetrical with respect to the sets and elements and (2) it extends the theorem to infinite families. For further generality to allow for the sets in the partition of the underlying set to be infinite, we state and prove the theorem in the "defect" form; that is, rather than requiring that the transversal intersect the partition within prescribed bounds, we require that the (set-theoretic) complement of the transversal do so.

THeOREM 3. Let $\mathfrak{A}(I)=\left(A_{i}: i \in I\right)$ be a family of subsets of a set $E$ and let $\overline{\mathfrak{A}}(E)=\left(\bar{A}_{e}: e \in E\right)$ be the dual family. Let $\sum_{k \in K} I_{k}$ and $\sum_{j \in \mathrm{J}} E_{j}$ be partitions of I and $E$ respectively. For each $k \in K$ let there be specified integers $c_{k}$ and $c_{k}^{\prime}$ with $0 \leqq c_{k} \leqq c_{k}^{\prime}$, and for each $j \in J$ let there be specified integers $d_{j}$ and $d_{j}^{\prime}$ with $0 \leqq d_{j} \leqq d_{j}^{\prime}$. Let $K^{\prime}$ $=\left\{k \in K: c_{k}^{\prime}<\left|I_{k}\right|\right\}$ and $J^{\prime}=\left\{j \in J: d_{j}^{\prime}<\left|E_{j}\right|\right\}$, and let $I^{*}=\bigcup_{k \in K^{\prime}} I_{k}$ and $E^{*}=\bigcup_{j \in J^{\prime}} E_{j}$. Assume for each $i \in I^{*}$ that $A_{i}$ is a finite set and for each $e \in E^{*}$ that $\bar{A}_{e}$ is a finite set. Let $K^{*}=\left\{k \in K: c_{k}>0\right\}$ and $J^{*}=\left\{j \in J: d_{j}>0\right\}$, and assume for each $k \in K^{*}$ that $I_{k}$ is a finite set and for each $j \in J^{*}$ that $E_{j}$ is a finite set.

Then there exists $I^{0} \subseteq I$ and $E^{0} \subseteq E$ such that $E^{0}$ is a transversal of $\mathfrak{A}\left(I^{0}\right)$ with

$$
\begin{array}{ll}
c_{k} \leqq\left|I_{k}\right| I^{0} \mid \leqq c_{k}^{\prime} & (k \in K) \\
d_{j} \leqq\left|E_{j}\right| E^{0} \mid \leqq d_{j}^{\prime} & (j \in J)
\end{array}
$$

if and only if the two conditions below are satisfied:

(3.3) For each finite subset $U$ of $I^{*}$ and for each finite subset $V$ of $J^{*}$,

$$
\left|\left(\bigcup_{i \in U} A_{i}\right) \cap\left(\bigcup_{j \in V} E_{j}\right)\right| \geqq|U|+\sum_{j \in V}\left(d_{j}-\left|E_{j}\right|\right)-\sum_{k: U \cap I_{k} \neq \varnothing} c_{k}^{\prime} .
$$

(3.4) For each finite subset $P$ of $E^{*}$ and for each finite subset $Q$ of $K^{*}$,

$$
\left|\left(\bigcup_{e \in P} \bar{A}_{e}\right) \cap\left(\bigcup_{k \notin Q} I_{k}\right)\right| \geqq|P|+\sum_{k \in Q}\left(c_{k}-\left|I_{k}\right|\right)-\sum_{j: P \cap E_{j} \neq \varnothing} d_{j}^{\prime}
$$

Proof. By Theorem 2 and the definition of the dual family there exists $I^{\circ} \subseteq I$ and $E^{0} \subseteq E$ such that $E^{0}$ is a transversal of $\mathscr{O}\left(I^{0}\right)$ with (3.1) and (3.2) valid if and only if both $\left(\mathrm{A}^{1}\right)$ and $\left(\mathrm{B}^{1}\right)$ below are true. 
$\left(\mathrm{A}^{1}\right)$ There exist $I^{\prime} \subseteq I$ and $E^{\prime} \subseteq E$ such that $E^{\prime}$ is a transversal of $\mathfrak{A}\left(I^{\prime}\right)$ with

$$
\begin{aligned}
& \left|I_{k} \backslash I^{\prime}\right| \leqq c_{k}^{\prime} \quad(k \in K) \\
& d_{j} \leqq\left|E_{j}\right| E^{\prime} \mid \quad(j \in J) .
\end{aligned}
$$

( $\left.\mathrm{B}^{1}\right)$ There exist $E^{\prime \prime} \subseteq E$ and $I^{\prime \prime} \subseteq I$ such that $I^{\prime \prime}$ is a transversal of $\overline{\mathfrak{A}}\left(E^{\prime \prime}\right)$ with

$$
\begin{array}{cl}
\left|E_{j}\right| E^{\prime \prime} \mid \leqq d_{j}^{\prime} & (j \in J) \\
c_{k} \leqq\left|I_{k} \backslash I^{\prime \prime}\right| & (k \in K) .
\end{array}
$$

We shall show that $\left(\mathrm{A}^{1}\right)$ is valid if and only if condition (3.3) is valid. By exploiting the symmetry of the situation it will follow that $\left(\mathrm{B}^{1}\right)$ is valid if and only if condition (3.4) is valid. This will prove the theorem.

Now if there exist $I^{\prime} \subseteq I$ and $E^{\prime} \subseteq E$ satisfying $\left(\mathrm{A}^{1}\right)$, let $\left(e_{i}: i \in I^{\prime}\right)$ be a system of distinct representatives of $\mathfrak{A}\left(I^{\prime}\right)$ with $E^{\prime}=\bigcup_{i \in I^{\prime}}\left\{e_{i}\right\}$. If $\bar{I}=I^{\prime} \cap I^{*}$ and $\bar{E}=\bigcup_{i \in I}\left\{e_{i}\right\}$, then $\bar{E}$ is a transversal of $\mathfrak{A}(\bar{I})$ with $\left|I_{k}\right| \bar{I}|=| I_{k}\left|I^{\prime}\right|$ for $k \in K^{\prime}$ and $\left|E_{j}\right| E^{\prime}|\leqq| E_{j}|\bar{E}|$ for $j \in J$. Thus $\left|I_{k}\right| \bar{I} \mid \leqq c_{k}^{\prime}$ for $k \in K^{\prime}$ and $d_{j} \leqq\left|E_{j}\right| \bar{E} \mid$ for $j \in J$. Conversely, if there exists $\bar{I} \subseteq I^{*}$ and $\bar{E} \subseteq E$ such that $\bar{E}$ is a transversal of $\mathfrak{A}(\bar{I})$ with $\left|I_{k}\right| \bar{I} \mid \leqq c_{k}^{\prime}$ for $k \in K^{\prime}$ and $d_{j} \leqq\left|E_{j}\right| E^{\prime} \mid$ for $j \in J$, then also $\left|I_{k}\right| \bar{I} \mid \leqq c_{k}^{\prime}$ for $k \in K \backslash K^{\prime}$ since for such $k$, $\left|I_{k}\right| \leqq c_{k}^{\prime}$. Thus by taking $I^{\prime}=\bar{I}$ and $E^{\prime}=\bar{E},\left(\mathrm{~A}^{1}\right)$ is true. Thus $\left(\mathrm{A}^{1}\right)$ is equivalent to

$\left(\mathrm{A}^{2}\right)$ There exist $\bar{I} \subseteq I^{*}$ and $\bar{E} \subseteq E$ such that $\bar{E}$ is a transversal of $\mathfrak{U}(\bar{I})$ with

$$
\begin{aligned}
\left|I_{k}\right| \bar{I} \mid \leqq c_{k}^{\prime} & \left(k \in K^{\prime}\right) \\
d_{j} \leqq\left|E_{j}\right| \bar{E} \mid & (j \in J) .
\end{aligned}
$$

We now show that $\left(\mathrm{A}^{2}\right)$ is equivalent to finding a transversal of a family of sets which we construct. Let $\left(F_{k}: k \in K^{\prime}\right)$ be a family of mutually disjoint sets indexed by $K^{\prime}$ with $\left(\bigcup_{k \in K^{\prime}} F_{k}\right) \cap E=\varnothing$ and $\left|F_{k}\right|=c_{k}^{\prime}$ for $k \in K^{\prime}$. Let $\left(L_{j}: j \in J^{*}\right)$ be a family of mutually disjoint sets indexed by $J^{*}$ with $\left(\bigcup_{j \in J^{*}} L_{j}\right) \cap I^{*}=\varnothing$ and $\left|L_{j}\right|=d_{j}$ for $j \in J^{*}$. Let $F=\bigcup_{k \in K^{\prime}} F_{k}$ and $L=\bigcup_{j \in J^{*}} L_{j}$ and consider the family $\mathscr{B}\left(I^{*} \cup L\right)$ $=\left(B_{i}: i \in I^{*} \cup L\right)$ of finite subsets of $E \cup F$ where

$$
B_{i}=A_{i} \cup F_{k} \quad \text { if } i \in I^{*} \text { with } i \in I_{k}
$$

and

$$
B_{i}=E_{j} \quad \text { if } i \in L \text { with } i \in L_{j} .
$$

Suppose $\mathscr{B}\left(I^{*} \cup L\right)$ has a system of distinct representatives $\left(x_{i}: i \in I^{*} \cup L\right)$ with corresponding transversal $X=\bigcup_{i \in I^{*} \cup L}\left\{x_{i}\right\}$. If $\bar{I}=\left\{i \in I^{*}: x_{i} \in A_{i}\right\}$, then $\left(x_{i}: i \in \bar{I}\right)$ is a system of distinct representatives of $\mathfrak{A}(\bar{I})$ and $\bar{E}=\bigcup_{i \in \bar{I}}\left\{x_{i}\right\}$ is a transversal of $\mathfrak{A}(\bar{I})$. Moreover since $\left|F_{k}\right|=c_{k}^{\prime}$ for $k \in K^{\prime}$ it follows that $\left|I_{k}\right| \bar{I} \mid \leqq c_{k}^{\prime}$ for $k \in K^{\prime}$; since $\left|L_{j}\right|=d_{j}$ for $j \in J^{*}$ it follows that $d_{j} \leqq\left|E_{j}\right| \bar{E} \mid$ for $j \in J^{*}$ and thus for $j \in J$. Conversely, if $\left(\mathrm{A}^{2}\right)$ is satisfied, it follows in the natural way that $\mathscr{B}\left(I^{*} \cup L\right)$ has a transversal. Hence $\left(\mathrm{A}^{2}\right)$ is equivalent to

$\left(\mathrm{A}^{3}\right)$ The family $\mathscr{B}\left(I^{*} \cup L\right)=\left(B_{i}: i \in I^{*} \cup L\right)$ of finite subsets of $E \cup F$ has a transversal. 
We are now in a position to apply Theorem 1 . Thus $\mathscr{B}\left(I^{*} \cup L\right)$ has a transversal if and only if

(3.11) For each finite subset $T$ of $I^{*} \cup L$

$$
\left|\bigcup_{i \in T} B_{i}\right| \geqq|T| .
$$

Let $U=T \cap I^{*}$ and $W=T \cap L$. Then (3.11) is equivalent to

(3.12) For each finite subset $U$ of $I^{*}$ and for each finite subset $W$ of $L$,

$$
\left|\left(\bigcup_{i \in U} B_{i}\right) \cup\left(\bigcup_{i \in W} B_{i}\right)\right| \geqq|U|+|W| .
$$

Now for $i \in U$ with $i \in I_{k}, B_{i}=A_{i} \cup F_{k}$ with $\left|F_{k}\right|=c_{k}^{\prime}$, and for $i \in W$ with $i \in L_{j}$, $B_{i}=E_{j}$. Thus (3.12) is equivalent to

(3.13) For each finite subset $U$ of $I^{*}$ and for each finite subset $W$ of $L$

$$
\begin{aligned}
\left|\left(\bigcup_{i \in U} A_{i}\right) \cap\left(\bigcup_{j: W \cap L_{j}=\varnothing} E_{j}\right)\right| & +\sum_{k: U \cap I_{k} \neq \varnothing} c_{k}^{\prime} \\
& +\sum_{j: W \cap L \neq \varnothing}\left|E_{j}\right| \geqq|U|+|W| .
\end{aligned}
$$

Now for $U$ fixed the value of the left side of the inequality in (3.13) depends only on whether $W \cap L_{j}=\varnothing$ or $W \cap L_{j} \neq \varnothing$ for each $j \in J^{*}$, while the value of the right side depends on the cardinality of $W$. Thus by replacing $W$ by $W^{\prime}=\bigcup_{j: W \cap L_{j} \neq \varnothing} L_{j}$, the value of the left side does not change while the value of the right side is $|U|+$ $\sum_{j: W \cap L_{j} \neq \varnothing} d_{j} \geqq|U|+|W|$. Thus (3.13) is equivalent to

(3.14) For each finite subset $U$ of $I^{*}$ and for each finite subset $V$ of $J^{*}$

$$
\left|\left(\bigcup_{i \in U} A_{i}\right) \cap\left(\bigcup_{j \notin V} E_{j}\right)\right|+\sum_{k: U \cap I_{k} \neq \varnothing} c_{k}^{\prime}+\sum_{j \in V}\left|E_{j}\right| \geqq|U|+\sum_{j \in V} d_{j} .
$$

Thus $\left(\mathrm{A}^{3}\right)$, and therefore $\left(\mathrm{A}^{1}\right)$, is equivalent to (3.14) which is equivalent to (3.3). As previously remarked, it now follows by symmetry that $\left(B^{1}\right)$ is equivalent to (3.4). This completes the proof of the theorem.

RemarK. Since $d_{j}=0$ for $j \in J \backslash J^{*}$, we may replace $J^{*}$ by $J$ in condition (3.3). Likewise we may replace $K^{*}$ by $K$ in condition (3.4). Also since $c_{k}^{\prime} \geqq\left|I_{k}\right|$ for $k \in K \backslash K^{\prime}$ we may replace $I^{*}$ by $I$ in (3.3). Likewise we may replace $E^{*}$ by $E$ in (3.4).

4. Special cases of the principal theorem. The principal theorem, Theorem 3, contains as special cases all theorems that we know which assert the existence of a transversal of a family of sets (or subfamily thereof) with or without additional properties. We shall indicate in this section some of these special cases. First we shall take Theorem 3 out of the "defect" form and put it into a less general but somewhat more direct form $\left({ }^{2}\right)$.

${ }^{2}$ ) It has recently come to our attention that Theorem 4 has been independently established by J. Folkman and D. R. Fulkerson (Subgraphs of bipartite and directed graphs, Rand report, April 1968). Their method of proof is quite different from ours. 
TheOrem 4. Let $\mathfrak{A}(I)=\left(A_{i}: i \in I\right)$ be a family of subsets of a set $E$ and let $\overline{\mathfrak{A}}(E)=\left(\bar{A}_{e}: e \in E\right)$ be the dual family. Let $\sum_{k \in K} I_{k}$ and $\sum_{j \in J} E_{j}$ be partitions of I and $E$ respectively into finite sets. For each $k \in K$ let there be specified integers $a_{k}$ and $a_{k}^{\prime}$ with $0 \leqq a_{k} \leqq a_{k}^{\prime}$ and for each $j \in J$ let there be specified integers $b_{j}$ and $b_{j}^{\prime}$ with $0 \leqq b_{j}$ $\leqq b_{j}^{\prime}$. Let $K^{\prime}=\left\{k \in K: a_{k}>0\right\}$ and $J^{\prime}=\left\{j \in J: b_{j}>0\right\}$, and let $I^{*}=\bigcup_{k \in K^{\prime}} I_{k}$ and $E^{*}=\bigcup_{j \in J^{\prime}} E_{j}$. Assume for each $i \in I^{*}$ that $A_{i}$ is a finite set and for each $e \in E^{*}$ that $\bar{A}_{e}$ is a finite set.

Then there exists $I^{0} \subseteq I$ and $E^{0} \subseteq E$ such that $E^{0}$ is a transversal of $\mathfrak{A}\left(I^{0}\right)$ with

$$
\begin{array}{ll}
a_{k} \leqq\left|I_{k} \cap I^{0}\right| \leqq a_{k}^{\prime} & (k \in K) \\
b_{j} \leqq\left|E_{j} \cap E^{0}\right| \leqq b_{j}^{\prime} & (j \in J)
\end{array}
$$

if and only if the following two conditions below are satisfied:

(4.3) For each finite subset $U$ of $I^{*}$ (or equivalently of I) and for each finite subset $V$ of $J$,

$$
\left|\left(\bigcup_{i \in U} A_{i}\right) \cap\left(\bigcup_{j \notin V} E_{j}\right)\right| \geqq|U|-\sum_{j \in V} b_{j}^{\prime}-\sum_{k: U \cap I_{k} \neq \varnothing}\left(\left|I_{k}\right|-a_{k}\right) .
$$

(4.4) For each finite subset $P$ of $E^{*}$ (or equivalently of $E$ ) and for each finite subset $Q$ of $K$,

$$
\left|\left(\bigcup_{e \in P} \bar{A}_{e}\right) \cap\left(\bigcup_{k \notin Q} I_{k}\right)\right| \geqq|P|-\sum_{k \in Q} a_{k}^{\prime}-\sum_{j: P \cap E_{j} \neq \varnothing}\left(\left|E_{j}\right|-b_{j}\right) .
$$

Theorem 4 is a direct consequence of Theorem 3 and the remark following its proof. It is not as general as Theorem 3, for in Theorem 4 all the $I_{k}$ and $E_{j}$ must be finite sets. In case we insist that $I^{0}=I$, that is, require a transversal for the whole family $\mathfrak{A}(I)$, then further simplification takes place. We state this as another theorem. It is the transfinite generalization of the Hoffman-Kuhn theorem [7].

THEOREM 5. Let $\mathfrak{A}(I)=\left(A_{i}: i \in I\right)$ be a family of finite subsets of a set $E$ and let $\overline{\mathfrak{A}}(E)=\left(\bar{A}_{e}: e \in E\right)$ be the dual family. Let $\sum_{j \in J} E_{j}$ be a partition of $E$ into finite sets. For each $j \in J$ let there be specified integers $b_{j}$ and $b_{j}^{\prime}$ with $0 \leqq b_{j} \leqq b_{j}^{\prime}$. Let $J^{\prime}=\left\{j: b_{j}>0\right\}$ and let $E^{*}=\bigcup_{j \in J^{\prime}} E_{j}$. Assume for each $e \in E^{*}$ that $\bar{A}_{e}$ is a finite set.

Then there exists $E^{0} \subseteq E$ such that $E^{0}$ is a transversal of $\mathfrak{U}(I)$ with

$$
b_{j} \leqq\left|E_{j} \cap E^{0}\right| \leqq b_{j}^{\prime} \quad(j \in J)
$$

if and only if the following two conditions below are satisfied:

(4.6) For each finite subset $U$ of $I$ and for each finite subset $V$ of $J$,

$$
\left|\left(\bigcup_{i \in U} A_{i}\right) \cap\left(\bigcup_{j \notin V} E_{j}\right)\right| \geqq|U|-\sum_{j \in V} b_{j}^{\prime} .
$$

(4.7) For each finite subset $P$ of $E^{*}$ (or equivalently of $E$ )

$$
\left|\bigcup_{e \in P} \bar{A}_{e}\right| \geqq|P|-\sum_{j: P \cap E_{j} \neq \varnothing}\left(\left|E_{j}\right|-b_{j}\right) .
$$


Theorem 5 follows from Theorem 4 by taking $K=I, I_{i}=\{i\}$ for each $i \in I$, and $a_{i}=a_{i}^{\prime}=1$ for each $i \in I$. Condition (4.6) follows directly from that of (4.3) by observing that $I^{*}=I$ here. Condition (4.4) becomes here: For each finite subset $P$ of $E^{*}$ and for each finite subset $Q$ of $I$,

$$
\left|\left(\bigcup_{e \in P} \bar{A}_{e}\right) \cap(I \backslash Q)\right|+|Q| \geqq|P|-\sum_{j: P \cap E_{j} \neq \varnothing}\left(\left|E_{j}\right|-b_{j}\right) .
$$

But for $P$ fixed, the value of the right side of the above inequality is fixed while the smallest value of the left side is $\left|\bigcup_{e \in P} A_{e}\right|$. Thus (4.4) reduces to (4.7).

If, in addition, $\mathfrak{X}(I)$ is a finite family and $E$ is a finite set, condition (4.7) can be put in terms of the original family $\mathfrak{A}(I)=\left(A_{i}: i \in I\right)$. For if we fix a subset $V$ of $J^{\prime}$, then condition (4.7) is equivalent to: For each $P \subseteq \bigcup_{j \in V} E_{j}$,

$$
\left|\bigcup_{e \in P} \bar{A}_{e}\right|+\sum_{j \in V}\left|E_{j}\right|-|P| \geqq \sum_{j \in V} b_{j} .
$$

This being true for all subsets $V$ of $J^{\prime}$. But by the König-Egerváry Theorem [10], [11] (we do not go into details here) the preceding inequality is equivalent to: For all $U \subseteq I$ and for all $V \subseteq J^{\prime}$,

$$
\left|\left(\bigcup_{i \in U} A_{i}\right) \cap\left(\bigcup_{j \in V} E_{j}\right)\right|+|I|-|U| \geqq \sum_{j \in V} b_{j} .
$$

But if this inequality is satisfied for all $V \subseteq J^{\prime}$, it is also satisfied for all $V \subseteq J$ since $b_{j}=0$ for $j \in J \backslash J^{\prime}$. Thus, under the assumption that $\mathfrak{A}(I)$ is a finite family of subsets of a finite set $E$, conditions (4.6) and (4.7) can be combined into the single condition

(4.8) For all $U \subseteq I$ and for all $V \subseteq J$,

$$
\left|\left(\bigcup_{i \in U} A_{i}\right) \cap\left(\bigcup_{j \in V} E_{j}\right)\right| \geqq \max \left\{|U|-\sum_{j \notin V} b_{j}^{\prime},|U|-|I|+\sum_{j \in V} b_{j}\right\} .
$$

Condition (4.8) is the one found by Hoffman and Kuhn [7].

Another special case of Theorem 4 is a theorem proved by Mirsky and Perfect [11] which is a transfinite generalization of a theorem of Mendelsohn and Dulmage [8].

THEOREM 6. Let $\mathfrak{A}(I)=\left(A_{i}: i \in I\right)$ be a family of subsets of a set $E$ and $\overline{\mathfrak{A}}(E)=$ $\left(A_{e}: e \in E\right.$ ) be the dual family. Let $I^{*} \subseteq I$ and $E^{*} \subseteq E$ be given and assume for each $i \in I^{*}$ that $A_{i}$ is a finite set and for each $e \in E^{*}$ that $\bar{A}_{e}$ is a finite set. Then there exists $I^{0}$ and $E^{0}$ with $I^{*} \subseteq I^{0} \subseteq I$ and $E^{*} \subseteq E^{0} \subseteq E$ such that $E^{0}$ is a transversal of $\mathfrak{A}\left(I^{0}\right)$ if and only if the two conditions below are satisfied:

(4.9) For each finite subset $U$ of $I^{*},\left|\bigcup_{i \in U} A_{i}\right| \geqq|U|$.

(4.10) For each finite subset $V$ of $E^{*},\left|\bigcup_{e \in V} \bar{A}_{e}\right| \geqq|V|$.

Several other known theorems can be deduced as special cases of Theorem 4. We do not continue in this direction any further. The reader is referred to the expository paper [10] by Mirsky and Perfect for additional theorems of this type. 
5. Systems of representatives. In this section we no longer insist that the representatives of a family of sets be distinct. More precisely, if $\mathfrak{A}(K)=\left(A_{k}: k \in K\right)$ is a family of subsets of a set $E$, then the family $\left(e_{k}: k \in K\right)$ of elements of $E$ is a system of representatives of $\mathfrak{A}(K)$ provided $e_{k} \in A_{k}$ for all $k \in K$. Recently Mirsky [9] obtained necessary and sufficient conditions that a family $\mathfrak{A}(K)=\left(A_{k}: k \in K\right)$ of subsets of a set $E$ (with suitable finiteness assumptions) have a system of representatives $\left(e_{k}: k \in K\right)$ such that the frequency of occurrence of each element $e$ of $E$ in this system of representatives (that is, $\left|\left\{k \in K: e_{k}=e\right\}\right|$ ) lies within prescribed bounds. We shall obtain from Theorem 4 a theorem which, in essence, symmetrizes Mirsky's theorem. We now make this precise. Following Mirsky, if $\left(C_{i}: i \in I\right)$ is a family of sets and $J \subseteq I$, then $C(J)=\bigcup_{i \in J} C_{i}$.

THEOREM 7. Let $\mathfrak{A}(K)=\left(A_{k}: k \in K\right)$ be a family of finite subsets of a set $E$. Let $\overline{\mathfrak{A}}(E)=\left(\bar{A}_{e}: e \in E\right)$ be the dual family and suppose each $\bar{A}_{e}$ is finite. For each $k \in K$ let integers $a_{k}$ and $a_{k}^{\prime}$ be given with $0 \leqq a_{k} \leqq a_{k}^{\prime}$, and for each $e \in E$ let integers $b_{e}$ and $b_{e}^{\prime}$ be given with $0 \leqq b_{e} \leqq b_{e}^{\prime}$. Then there exists a family $\subseteq(K)=\left(S_{k}: k \in K\right)$ of subsets of $E$ with dual family $\bar{\Im}(E)=\left(\bar{S}_{e}: e \in E\right)$ such that $S_{k} \subseteq A_{k}$ for all $k \in K$ and thus $\bar{S}_{e} \subseteq \bar{A}_{e}$ for all $e \in E$ with

$$
\begin{array}{ll}
a_{k} \leqq\left|S_{k}\right| \leqq a_{k}^{\prime} & (k \in K) \\
b_{e} \leqq\left|\bar{S}_{e}\right| \leqq b_{e}^{\prime} & (e \in E)
\end{array}
$$

if and only if the two conditions below are satisfied:

(5.3) For each finite subset $X$ of $K$,

$$
\sum_{e \in A(X)} \min \left\{\left|\bar{A}_{e} \cap X\right|, b_{e}^{\prime}\right\} \geqq \sum_{k \in X} a_{k} .
$$

(5.4) For each finite subset $Y$ of $E$,

$$
\sum_{k \in A^{(}(Y)} \min \left\{\left|A_{k} \cap Y\right|, a_{k}^{\prime}\right\} \geqq \sum_{e \in Y} b_{e}
$$

Proof. For each $k \in K$, let $I_{k}=\left\{(k, e): e \in A_{k}\right\}$ so that $\left|I_{k}\right|=\left|A_{k}\right|$. Let $I=\bigcup_{k \in K} I_{k}$ so that $\sum_{k \in K} I_{k}$ is a partition of $I$. For each $e \in E$, let $F_{e}=\left\{(e, k): k \in \bar{A}_{e}\right\}$ so that $\left|F_{e}\right|=\left|\bar{A}_{e}\right|$. Let $F=\bigcup_{e \in E} F_{e}$ so that $\sum_{e \in E} F_{e}$ is a partition of $F$. Let $\sigma: I \rightarrow F$ be a bijection such that if $(k, e) \in I_{k}$ then $\sigma((k, e)) \in F_{e}$. (By well-ordering $K$ certainly such a bijection can be defined.) Let $\mathscr{B}(I)=\left(B_{i}: i \in I\right)$ be the family of one element subsets of $F$ defined by $B_{i}=\{\sigma(i)\}$ for all $i \in I$. Then there exists a family $\Im(K)$ $=\left(S_{k}: k \in K\right)$ of subsets of $E$ satisfying the conclusion of the theorem if and only if there exists $I^{0} \subseteq I$ and $F^{0} \subseteq F$ such that $F^{0}$ is a transversal of $\mathscr{B}\left(I^{0}\right)$ with

$$
\begin{array}{ll}
a_{k} \leqq\left|I_{k} \cap I^{0}\right| \leqq a_{k}^{\prime} & (k \in K) \\
b_{e} \leqq\left|F_{e} \cap F^{0}\right| \leqq b_{e}^{\prime} & (e \in E) .
\end{array}
$$


For, if there exists such a family $\mathfrak{s}(K)=\left(S_{k}: k \in K\right)$, take

$$
I^{0}=\bigcup_{k \in K}\left(\bigcup_{e \in S_{k}}(k, e)\right) \text { and } F^{0}=\bigcup_{k \in K}\left(\bigcup_{e \in S_{k}} \sigma((k, e))\right) .
$$

Then surely $F^{0}$ is a transversal of $\mathscr{B}\left(I^{0}\right)$. Since $S_{k}$ satisfies (5.1), $I^{0}$ satisfies (5.5). Also since $\left|F_{e} \cap F^{0}\right|=\left|\bar{S}_{e}\right|$ and $\bar{S}_{e}$ satisfies (5.2), $F^{0}$ satisfies (5.6). Conversely, if there exists $I^{0} \subseteq I$ and $F^{0} \subseteq F$ such that $F^{0}$ is a transversal of $\mathscr{B}\left(I^{0}\right)$ with (5.5) and (5.6) valid, then for each $k \in K$ let $S_{k}=\left\{e \in A_{k}:(k, e) \in I_{k} \cap I^{0}\right\}$. It follows that $\left|S_{k}\right|$ $=\left|I_{k} \cap I^{0}\right|$ for all $k \in K$ and that $\left|\bar{S}_{e}\right|=\left|F_{e} \cap F^{0}\right|$ for all $e \in E$. Thus $\mathfrak{S}(K)=$ $\left(S_{k}: k \in K\right)$ is a family of subsets of $E$ with $S_{k} \subseteq A_{k}$ for all $k \in K$ such that $\subseteq(K)$ and its dual $\overline{\mathfrak{S}}(E)$ satisfy (5.1) and (5.2).

We are now in a position to apply Theorem 4 to the family $\mathscr{B}(I)$ of subsets of $F$. Thus there exists $I^{0} \subseteq I$ and $F^{0} \subseteq I$ such that $F^{0}$ is a transversal of $\mathscr{B}\left(I^{0}\right)$ with (5.5) and (5.6) valid if and only if the following two conditions are true:

(5.7) For each finite subset $U$ of $I$ and for each finite subset $V$ of $E$,

$$
\left|\left(\bigcup_{i \in U} B_{i}\right) \cap\left(\bigcup_{e \notin V} F_{e}\right)\right|+\sum_{e \in V} b_{e}^{\prime} \geqq|U|-\sum_{k: U \cap I_{k} \neq \varnothing}\left(\left|I_{k}\right|-a_{k}\right) .
$$

(5.8) For each finite subset $P$ of $F$ and for each finite subset $Q$ of $K$,

$$
\left|\left(\bigcup_{f \in P} \bar{B}_{f}\right) \cap\left(\bigcup_{k \notin Q} I_{k}\right)\right|+\sum_{k \in Q} a_{k}^{\prime} \geqq|P|-\sum_{e: P \cap F_{e} \neq \varnothing}\left(\left|F_{e}\right|-b_{e}\right) .
$$

Consider condition (5.7) with $V$ fixed. Let $U$ be a finite subset of $I$ and let $U^{\prime}$ $=\bigcup_{k: U \cap I_{k} \neq \varnothing} I_{k}$. If (5.7) is satisfied with $U$ replaced by $U^{\prime}$, then since the $B_{i}$ are one element subsets of $F,(5.7)$ will also be satisfied for $U$. Thus (5.7) is equivalent to

(5.9) For each finite subset $X$ of $K$ and for each finite subset $V$ of $E$,

$$
\sum_{e \notin V}\left|\bar{A}_{e} \cap X\right|+\sum_{e \in V} b_{e}^{\prime} \geqq \sum_{k \in X} a_{k} .
$$

But for $X$ fixed, the minimum of the left side of the previous inequality is

$$
\sum_{e \in A(X)} \min \left\{\left|\bar{A}_{e} \cap X\right|, b_{e}^{\prime}\right\}
$$

Thus (5.9) and therefore (5.7) is equivalent to (5.3). Similarly (5.8) is equivalent to (5.4). This completes the proof of the theorem.

In proving Theorem 7 we have not used the full strength of Theorem 4, for we have assumed all the sets $A_{k}$ and all the sets $\bar{A}_{e}$ are finite. Thus a somewhat stronger theorem can be proved in which those $A_{k}$ with $a_{k}=0$ and those $\bar{A}_{e}$ with $b_{e}=0$ are not assumed to be finite. However the notation to derive such a result from Theorem 4 is rather formidable, and we forego the details.

Corollary. Let $\mathfrak{A}(K)=\left(A_{k}: k \in K\right)$ be a family of finite subsets of a set $E$ and suppose each $e \in E$ is an element of only finitely many $A$ 's. For each $e \in E$ let integers 
$b_{e}$ and $b_{e}^{\prime}$ be given. Then $\mathfrak{A}(K)$ has a system of representatives $\left(e_{k}: k \in K\right)$ such that

$$
b_{e} \leqq\left|\left\{k: e_{k}=e\right\}\right| \leqq b_{e}^{\prime} \quad(e \in E)
$$

if and only if the two conditions below are valid:

(5.10) For each finite subset $X$ of $K$,

$$
\sum_{e \in A(X)} \min \left\{\left|\left\{k \in X: e \in A_{k}\right\}\right|, b_{e}^{\prime}\right\} \geqq|X| .
$$

(5.11) For each finite subset $Y$ of $E$,

$$
\left|\left\{k \in K: A_{k} \cap Y \neq \varnothing\right\}\right| \geqq \sum_{e \in Y} b_{e} .
$$

Proof. Since each $e \in E$ is an element of only finitely many $A$ 's, each set $\bar{A}_{e}$ in the dual family $\overline{\mathfrak{A}}(E)=\left(\bar{A}_{e}: e \in E\right)$ of $\mathfrak{U}(I)$ is a finite set. The corollary then follows by defining $a_{k}=a_{k}^{\prime}=1$ and applying Theorem 7.

In [9] Mirsky proves a similar theorem (he allows those $e$ with $b_{e}=0$ to be elements of infinitely many $A$ 's), but while he obtains condition (5.11), his condition corresponding to (5.10) is different. More precisely, in Mirsky's theorem (5.10) is replaced with

(5.12) For each finite subset $X$ of $K, \sum_{e \in A(X)} b_{e}^{\prime} \geqq|X|$.

It follows therefore that (5.10) is equivalent to (5.12).

6. The subgraph problem for directed graphs. A directed graph $D=(P, L)$ consists of a set $P$ the elements of which are called points and a set $L$ of ordered pairs of points which are called lines [6]. If $p$ and $q$ are points and $l=(p, q)$ is a line, then $l$ is said to be a line from $p$ to $q$. If $X \subseteq P$, then

$$
A(X)=\{q \in P:(p, q) \in L \text { for some } p \in X\}
$$

and

$$
B(X)=\{q \in P:(q, p) \in L \text { for some } p \in X\} .
$$

If $X$ consists of a single point $p$, then we shall identify $A(\{p\})$ and $B(\{p\})$ with $A(p)$ and $B(p)$ respectively. The outdegree of a point $p, \operatorname{od}_{D}(p)=|A(p)|$, the number of lines from $p$; the indegree of $p, \operatorname{id}_{D}(p)=|B(p)|$, the number of lines to $p$. A (spanning) subgraph of a directed graph $(P, L)$ is a directed graph $D^{\prime}=\left(P, L^{\prime}\right)$ with $L^{\prime} \subseteq L$. The subgraph problem for directed graphs asks for necessary and sufficient conditions that a directed graph have a subgraph with prescribed indegrees and outdegrees for each of its points. This was solved for finite directed graphs (that is, directed graphs with a finite number of points) by Ore [12]. In their book [3] Ford and Fulkerson gave and solved an extension of this problem for finite directed graphs. Their problem was that given a finite directed graph find necessary and sufficient conditions that there exist a subgraph such that the indegrees and outdegrees of the points lie within prescribed bounds. We shall extend the theorem of Ford and 
Fulkerson to locally finite directed graphs. A directed graph $D=(P, L)$ is locally finite provided the indegrees and outdegrees of each of its points are finite.

THEOREM 8. Let $D=(P, L)$ be a locally finite directed graph. Corresponding to each $p \in P$ let there be prescribed integers $a_{p}, a_{p}^{\prime}, b_{p}, b_{p}^{\prime}$ satisfying

$$
0 \leqq a_{p} \leqq a_{p}^{\prime}, \quad 0 \leqq b_{p} \leqq b_{p}^{\prime} \quad(p \in P) .
$$

Then $D$ has a subgraph $D^{\prime}$ with

$$
\begin{array}{ll}
a_{p} \leqq \operatorname{id}_{D^{\prime}}(p) \leqq a_{p}^{\prime} & (p \in P) \\
b_{p} \leqq \operatorname{od}_{D^{\prime}}(p) \leqq b_{p}^{\prime} & (p \in P)
\end{array}
$$

if and only if for all finite subsets $X$ of $P$ the following two conditions are satisfied:

$$
\begin{gathered}
\sum_{q \in A(X)} \min \left\{|B(q) \cap X|, b_{q}^{\prime}\right\} \geqq \sum_{p \in X} a_{p} \\
\sum_{q \in B(X)} \min \left\{|A(q) \cap X|, a_{q}^{\prime}\right\} \geqq \sum_{p \in X} b_{p} .
\end{gathered}
$$

Proof. Let $\mathfrak{A}(P)=\left(A_{p}: p \in P\right)$ be a family of subsets of $P$ defined by $A_{p}=A(p)$ $=\{q \in P:(p, q) \in L\}$. The dual family $\overline{\mathfrak{A}}(P)=\left(\bar{A}_{p}: p \in P\right)$ satisfies $\bar{A}_{p}=B(p)$. Since the directed graph $D$ is locally finite, for each $p \in P$ both $A_{p}$ and $\bar{A}_{p}$ are finite sets. It is clear that the directed graph $D$ has a subgraph $D^{\prime}$ satisfying (6.1) and (6.2) if and only if there exists a family $\subseteq(P)=\left(S_{p}: p \in P\right)$ of subsets of $P$ with dual family $\bar{\Xi}(P)=\left(\bar{S}_{p}: p \in P\right)$ such that $S_{p} \subseteq A_{p}$ and $\bar{S}_{p} \subseteq \bar{A}_{p}$ with

$$
a_{p} \leqq\left|S_{p}\right| \leqq a_{p}^{\prime}, \quad b_{p} \leqq\left|\bar{S}_{p}\right| \leqq b_{p}^{\prime} \quad(p \in P) .
$$

The theorem now follows by applying Theorem 7 .

In case $D$ is a finite directed graph, Theorem 8 reduces to the theorem of Ford and Fulkerson. If also $a_{p}=a_{p}^{\prime}$ and $b_{p}=b_{p}^{\prime}$ for all $p \in P$, then Theorem 8 reduces to the theorem of Ore. For $D$ a locally finite directed graph, if $a_{p}=a_{p}^{\prime}$ and $b_{p}=b_{p}^{\prime}$ for all $p \in P$, then Theorem 8 describes when $D$ has a subgraph for which the indegree and outdegree of each point $p$ is $a_{p}$ and $b_{p}$ respectively.

\section{REFERENCES}

1. S. Banach, Un théorème sur les transformations biunivoques, Fund. Math. 6 (1924), 236-239

2. R. A. Brualdi, An extension of Banach's mapping theorem, Proc. Amer. Math. Soc. 20 (1969), 520-526.

3. L. R. Ford, Jr. and D. R. Fulkerson, Flows in networks, Princeton Univ. Press, Princeton, N. J., 1962.

4. M. Hall, Jr., Distinct representatives of subsets, Bull. Amer. Math. Soc. 54 (1948), 922-926.

5. P. Hall, On representatives of subsets, J. London Math. Soc. 10 (1935), 26-30.

6. F. Harary, R. Z. Norman and D. Cartwright, Structural models, Wiley, New York, 1965.

7. A. J. Hoffman and H. W. Kuhn, On systems of distinct representatives, Annals of Mathematical Studies No. 38, Princeton Univ. Press, Princeton, N. J., 1956, pp. 199-206. 
8. N. S. Mendelsohn and A. L. Dulmage, Some generalizations of the problem of distinct representatives, Canad. J. Math. 10 (1958), 230-241.

9. L. Mirsky, Systems of representatives with repetition, Proc. Cambridge Philos. Soc. 63 (1967), 1135-1140.

10. L. Mirsky and H. Perfect, Systems of representatives, J. Math. Anal. Appl. 15 (1966), 520-568.

11. - Applications of the notion of independence to problems of combinatorial analysis, J. Combinatorial Theory 2 (1967), 327-357.

12. O. Ore, Studies on directed graphs. I, Ann. of Math. (2) 63 (1956), 383-406.

UNIVERSITY OF WISCONSIN,

Madison, Wisconsin 